\title{
Group-Mobility-Aware Spectrum Management for Future Digital Battlefields
}

\author{
Heather (Haitao) Zheng \\ Department of Computer Science \\ University of California, Santa Barbara \\ htzheng@cs.ucsb.edu
}

\author{
Juwei Shi \\ Beijing Univ. of Posts and Telecom., \\ Beijing, P.R. China \\ shijuwei@gmail.com
}

\author{
Lili Cao \\ Dept. of Computer Science \\ University of California, Santa Barbara \\ cao_li_li@yahoo.com
}

\begin{abstract}
Efficient access to spectrum is critical to maintain reliable and robust communication in military networks, especially for ground vehicles on the move. High mobility and frequent topology variations make spectrum management in battlefields challenging. In this paper, we present a decentralized, hierarchical approach to assign spectrum channels among vehicles in a fair and efficient manner. Making use of group mobility, we propose a low-complexity allocation scheme with minimum communication and computation overhead. Experimental results demonstrate that the proposed algorithm can reduce the overhead by $10+$ fold with maintaining reasonable spectrum utilization.
\end{abstract}

\section{INTRODUCTION}

Spectrum management assigns spectrum resource across users to prevent interference and contention, while promoting utilization and fairness. For static or slow varying networks, existing solutions apply centralized graph coloring algorithms [9], [13], and decentralized coordinations [3], [12] to approximate the optimal assignment.

In highly mobile networks such as digital battlefields, users travel at high speeds and network topologies change frequently, making spectrum management a great challenge. Using centralized approaches, the server needs to repeatedly perform assignment when conflict topology changes, incurring large computation and communication overhead. Similar limitations apply to existing systems based on decentralized coordination [3], [12], where the overhead scales linearly with the ratio of mobile nodes in the network. To efficiently manage spectrum usage in battlefields with networkwide mobility, we need to develop new low-complexity algorithms that quickly adapt to topology variations.

While high mobility makes the problem of spectrum management challenging, we observe, however, that it is not a fundamental limitation. User mobility in battlefields follows a group mobility pattern. Users travel in groups at high speeds, but each group maintains a relatively stable topology. The patterns of group mobility have been verified and modeled by extensive studies [2], [4], [14]. It provides great potential to build a low-cost and highly-adaptive spectrum management system.

In this work, we make use of group mobility patterns and propose a hierarchial spectrum allocation approach to distribute spectrum channels among mobile combat vehicles. When their movements lead to variations in conflict conditions, vehicles exercise a novel distributed approach based on inter-group and intra-group coordinations. Our approach has a hierarchical structure where only a few selected vehicles from different mobile groups perform inter-group coordinations to divide spectrum coarsely among groups. After this is done, the majority of vehicles only apply a simple mapping to adjust their channel usage to the new group-level assignments and selectively invoke intra-group coordinations to resolve any sub-optimality introduced by the mapping. Through the proposed multi-stage coordinations, our system provides quickly approximates the new optimal assignment.

The decentralized, hierarchical design makes our proposed approach low-cost and simple to implement. Our system does not require any central server and the coordinations are implemented as message exchanges among individual vehicles. Different from prior work on device coordination [3], the majority of vehicles only interact with their peers from the same group. Since each group has a relatively stable topology, vehicles can quickly adapt based on their prior assignments, significantly reducing the occurrence of coordinations. Having a stable topology also promotes reliable message transmissions even under high group mobility. Finally, by partitioning spectrum among groups based on predicted conflict conditions, our approach minimizes the occurrence of inter-group coordinations.

We perform experiments to examine the performance of the proposed approach, while comparing it to the prior 
work [3] that does not consider group mobility. Using a representative scenario where two mobile groups travel across a battlefield, we show that the proposed approach significantly reduces computation and communication overhead, by $10-50$ fold, while maintaining reasonable spectrum utilization.

The rest of the paper is organized as follows. We begin in Section II by briefly describing existing solutions on spectrum allocation and the impact of group mobility on these solutions. Next in Section III, we propose a hierarchical coordination framework and describe the detailed system design. In Section IV, we conduct experiments to evaluate the performance of different spectrum allocation approaches. Finally, we discuss implications and future directions, and conclude in Section V.

\section{BACKGROUND}

In this section, we briefly describe existing solutions on spectrum management and the implications of group mobility on these solutions.

\section{A. Spectrum Management in Digital Battlefields}

Spectrum management allocates spectrum usage across wireless devices to prevent interference, while promoting utilization and fairness. Channels are the fundamental units of spectrum usage. The operational spectrum is partitioned into orthogonal channels by one or a combination of time-, frequency-, or code-division. While many efforts have focused on adjusting transmit power to reduce interference, spectrum management focuses on the use of channel assignment to achieve conflict-free transmissions at fixed transmit power.

Efficient spectrum management is critical to maintain reliable communication in digital battlefields. Combat soldiers and vehicles need to frequently interact with their peers, through soldier-to-vehicle, vehicle-tosoldier, or helicopter-to-soldier communications. Concurrent transmissions in physical proximity could conflict with each other and should operate on orthogonal channels to prevent interference.

Using a simple scenario where soldiers communicate with vehicles, we now describe the problems and challenges facing spectrum management. In this scenario, each vehicle is an access point for ground soldiers, and uses a set of channels to communicate with its affiliated soldiers. While each vehicle can apply existing MAC protocols such as TDMA, FDMA, CSMA to resolve contentions among its affiliated soldiers, their transmissions still suffer from interference due to transmissions at nearby vehicles and soldiers. One key goal of spectrum management is to assign orthogonal channels to conflicting ${ }^{1}$ vehicles to prevent this type of interference. In addition, efficient spectrum allocation also promotes spectrum utilization and user fairness.

\section{B. Existing Spectrum Management Solutions}

Existing solutions on spectrum management focus on optimizing channel assignment to maximize system utility. Combinations of spectrum utilization and user fairness form specific system utility functions that can be customized for different types of network applications. These include proportional fairness [7], [9], minimum node throughput and total system throughput [6].

Optimal conflict-free channel assignment towards a global optimal system utility is in general NP-hard [5], [6]. Both centralized and decentralized approximations have been proposed for static or slow-varying networks. Centralized approaches reduce the problem into a graphcoloring problem [1], [5], [6], [9] and focus on heuristics based approximations performed at a central server. Alternative approaches apply device coordination to resolve conflict and refine channel assignment locally, significantly reducing communication and computation complexity without relying on any central servers. Both explicit [3], [8] and implicit [12] coordination approaches have been proposed to quickly adapt to small changes affecting a local network area.

\section{Group Mobility and Its Implications.}

Studies observe strong group mobility patterns at mobile users in real military networks [2], [4], [14]. Users in the same mission travel together following a similar track. They form mobile groups and the speed of internal mobility within a group is much less than the speed of group movement.

High mobility at individual users leads to fast variations in interference constraints among neighboring peers. In Figure 1 we illustrate this effect by modeling the interference constraints using a conflict graph. Each vertex represents a mobile vehicle serving as an access point for ground soldiers (we ignore the soldiers to simplify the graph), and an edge between two vertices if they conflict. Assuming two mobile groups travel

\footnotetext{
${ }^{1} \mathrm{We}$ assume that each vehicle implements a conflict discovery mechanism to identify conflicting peers. For example, they can perform signalling based detection by periodically broadcasting high power beacons and recognizing a conflicting peer by its beacon signal strength [8].
} 


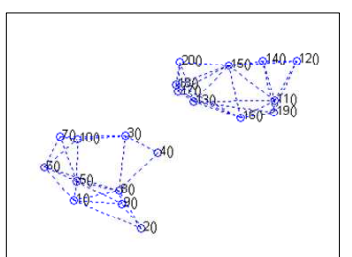

(a) Topology in Timeslot 1

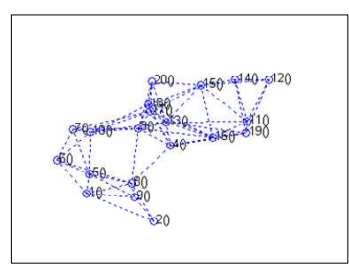

(b) Topology in Timeslot 7

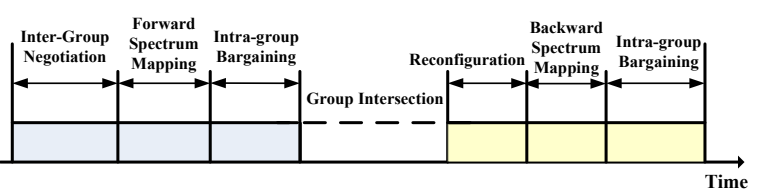

Fig. 3. The timeline of the proposed hierarchical coordination system.

Fig. 1. An example conflict graph representing interference constraints before and during group intersection. Each vertex represents a vehicle at its physical location, an edge exists between two nodes if they conflict. (a) Topology when groups are well-separated. (b) Topology when groups intersect.

towards each other, we show that the conflict graphs at time slot 0 and 7 differ greatly.

Existing spectrum management approaches [3] do not consider group mobility. Users need to frequently adapt their spectrum usage by coordinating with conflicting peers from the same and different groups. In Figure 2 we plot the total number of messages exchanged in the network, and the number of users involved in order to approach a new optimal assignment in each time slot. A significant ratio $(70+\%)$ of users are involved, incurring high communication overhead and long disruptions to transmissions.

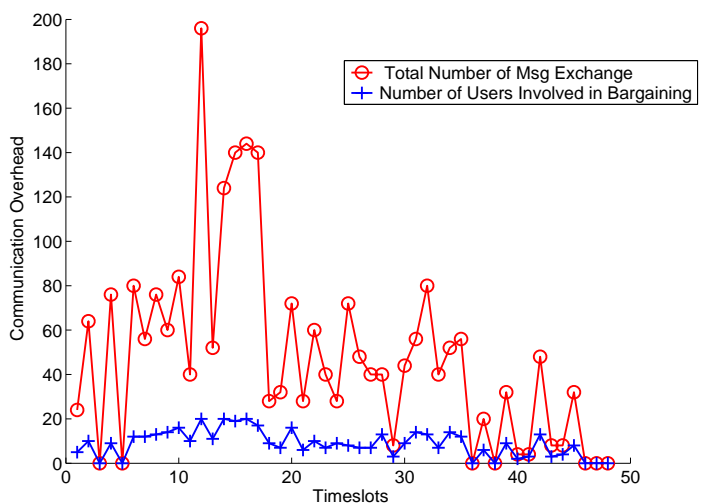

Fig. 2. Message overhead of the conventional coordination approach [3]. Two groups (10 vehicles each) travel at $30 \mathrm{~km} / \mathrm{h}$ towards each other. In each time slot, the network-wide conflict graph changes, and vehicles coordinate to approach a new conflict-free spectrum allocation.

\section{HiERARCHICAL BARGAINING FrAMEWORK}

To reduce the coordination overhead observed in Figure 2 we need a new spectrum management approach that treats complexity and overhead minimization as the first priority. To address this challenge, we propose a hierarchical coordination approach that takes advantage of group mobility. We focus on the scenario described in Section II-A where combat vehicles serve as access points for ground soldiers. The problem of spectrum management is to assign channels to vehicles to prevent conflicts and maximize system utility. Each vehicle is equipped with a wireless radio device that is capable of communicating on multiple non-continuous channels. The notions of device and vehicle are interchangeable.

\section{A. System Overview}

Group mobility results in a hierarchical structure in network topology and conflict graph. While each mobile group moves at high speeds, its local group topology and conflict graph remain relatively stable. When two mobile groups start to conflict, each device adjusts its channel usage in two stages. In the first stage, spectrum usage is divided among groups to prevent inter-group interference. In the second stage, devices in each group perform local adjustments to approach the group-level optimal assignment. The majority of devices only participate in the second stage and interact with peers from the same group. Since each group has a relatively stable topology, this can effectively minimize the occurrence of coordination in the presence of high mobility.

The detailed implementation consists of the following design components:

- Inter-group negotiation. Several selected users (those located at the group edges) perform negotiations to partition spectrum across multiple groups.

- Spectrum mapping. When a group's spectrum usage changes, its members perform a simple mapping to quickly transform their existing spectrum usage to the new group-level assignment.

- Intra-group negotiation. Upon detecting a suboptimal assignment due to the simple mapping, users within each group perform local adjustments by coordinating with peers from the same group.

In this paper, our work is driven by a simple scenario ${ }^{2}$ where two mobile groups travel across each other and the corresponding network conflict-graph changes frequently

\footnotetext{
${ }^{2}$ This is not a limitation of our work since our approach can be easily extended to other scenarios.
} 
(see Figure 1). When well-separated, each group has full access to spectrum channels. During the time they intersect and conflict, channels are divided between the groups. The use of spectrum mapping and intra-group negotiation provides quick adaptation to not only variations in group-level spectrum assignment, but also variations in group topology due to internal mobility. Figure 3 illustrates the timeline of the proposed approach. Next, we describe these components in detail.

\section{B. Inter-Group Spectrum Negotiation}

As two mobile groups move towards each other, devices from different groups start to conflict. To prevent this type of conflict, we propose to separate usable channels between the groups. As groups travel away from each other and become non-conflicting, devices can gradually expand their channel usage.

Our proposed inter-group negotiation is decentralized and does not rely on any central server. Each group selects a member as the negotiator. The negotiator selection is automatic and distributed. When detecting a conflicting vehicle from a different group, a device broadcasts an alarm message to its group members and becomes the inter-group negotiator. It contacts the conflicting neighbor from the other group to perform the negotiation $^{3}$

The negotiators coordinate to partition spectrum usage among groups. The research challenge is how to derive a fair and efficient spectrum partition that closely approximates the global optimal solution. We propose a povertyline driven spectrum partition. Poverty-line, proposed in [3], represents the minimum number of channels a device can obtain through coordinations. If a group $i$ is assigned with $L_{i}$ channels, then a vehicle $n$ in group $i$, if surrounded by $D_{n, i}$ conflicting peers in the group, will have a poverty line $P L(n, i)$ as

$$
P L(n, i)=\left\lfloor\frac{L_{i}}{D_{n, i}+1}\right\rfloor .
$$

To achieve fairness, we propose to equalize the average group poverty line. For any two conflicting groups $i$ and $j$, this means

$$
E_{n \in i}\left\{\left\lfloor\frac{L_{i}}{D_{n, i}+1}\right\rfloor\right\}=E_{n \in j}\left\{\left\lfloor\frac{L_{j}}{D_{n, j}+1}\right\rfloor\right\} .
$$

\footnotetext{
${ }^{3}$ We assume users operate on a globally-known common control channel [10], [11] to communicate with peers from different groups. While the efficiency of the negotiation depends on the underlying protocol, it is not the main focus of this paper. We will address the protocol design in a detailed study.
}

If $L_{i}>>D_{n, i}, L_{j}>>D_{n, j}$, we can approximate the constraint as

$$
\frac{L_{i}}{L_{j}}=\frac{E_{n \in j}\left\{\frac{1}{D_{n, j}+1}\right\}}{E_{n \in i}\left\{\frac{1}{D_{n, i}+1}\right\}} .
$$

Each device needs to keep track of the average conflict level $E_{n \in i}\left\{\frac{1}{D_{n, i}+1}\right\}$ in its group, and the distribution of channel usage. Negotiators can use these information to select channels used by the majority of its group members and minimize the impact of spectrum partition.

The proposed inter-group spectrum partition can significantly reduce the coordination overhead. Users from different groups do not perform coordinations in each time slot but only at the first time slot when they start to intersect. However, this partition could lead to suboptimal spectrum utilization, since it is based on the worst case assumption that users from different groups always conflict with each other.

\section{Simple Spectrum Mapping}

After inter-group coordination, negotiators broadcast changes in group-level channel assignments to its group members. Devices need to adjust their channel usage to prevent conflict and promote utilization and fairness. One approach is to perform group-wide coordination assuming no prior assignment information, which incurs high computation and communication overhead. To reduce these overheads, we propose the use of a simple spectrum mapping that takes prior assignment into account when deriving new assignment.

There are two types of mapping. When groups intersect, their channel usages shrink and devices perform a forward-mapping. Once groups become well-separated and non-conflicting, devices perform a backwardmapping to expand their channel usage. Let $\mathbb{L}_{i}$ and $\mathbb{L}_{i}^{\prime}$ represent the channel sets assigned to group $i$ before and after the inter-group negotiation, respectively. Let $\left|\mathbb{L}_{i}\right|=L_{i},\left|\mathbb{L}_{i}^{\prime}\right|=L_{i}^{\prime}$.

- Forward-mapping $\mathfrak{F}\left(a \rightarrow b, a \in \mathbb{L}_{i}, b \in \mathbb{L}^{\prime}{ }_{i}\right)$.

In this case, $L_{i} \geq L_{i}^{\prime}$ and $\mathfrak{F}$ is a many-to-one mapping. A naive design can produce interference when two conflicting devices map different channels to a single channel in the new set. To avoid this, we introduce a "NULL" channel into $\mathbb{L}_{i}^{\prime}$ and map $L_{i}-L_{i}^{\prime}$ channels to the NULL channel.

- Backward Mapping $\mathfrak{B}\left(a \rightarrow b, a \in \mathbb{L}_{i}, b \in \mathbb{L}^{\prime}{ }_{i}\right)$. $L_{i} \leq L_{i}^{\prime}$ and $\mathfrak{B}$ is a simple one-to-many mapping, i.e. $\mathfrak{B}\left(a \rightarrow\left(b_{1}, b_{2}, \ldots, b_{k}\right), a \in \mathbb{L}_{i}, b_{1}, b_{2}, \ldots, b_{k} \in\right.$ $\left.\mathbb{L}_{i}^{\prime}\right)$, and $k=\left\lfloor\frac{L_{i}^{\prime}}{L_{i}}\right\rfloor$. 


\section{Intra-Group Coordination via Local Bargaining}

The spectrum mapping is coarse and could lead to sub-optimal channel assignments. Affected devices perform intra-group coordination to refine assignments by coordinating with peers from the same group. The detailed bargaining procedure is described in [3]. Since the goal of intra-group coordination is to refine channel assignments, this approach incurs much lower overhead compared to that of the group-wide coordination without considering any prior assignment.

While simple and low-cost, our approach offers hard guarantee on individual device's channel usage. Assuming that the channels are partitioned between groups to equalize their average poverty line, the proposed approach guarantees a poverty line for user $n$ at group $i$ as follows:

$$
P L(n, i)=\left\lfloor\frac{L \cdot E_{m \in i}\left\{\frac{1}{D_{m, i}+1}\right\}}{\sum_{j=1}^{J} E_{m \in j}\left\{\frac{1}{D_{m, j}+1}\right\}} \cdot \frac{1}{D_{n, i}+1}\right\rfloor,
$$

where $L$ represents the total number of channels available for the entire networks, and $J$ represents the total number of groups conflicting with group $i$, including $i$ itself. Devices can use their poverty line to initiate coordinations - a device whose channel assignment is less than its poverty line generates requests to its peers to improve its channel usage.

\section{EXPERIMENTAL RESULTS}

\section{A. Simulation Setup and Performance Metrics}

We perform experiments to examine the performance of different allocation schemes. We simulate a random network on a $250 m \times 250 m$ area. Each node represents a combat vehicle and supports communications with its affiliated soldiers. Using a simple protocol based interference model [5], we assume two vehicles conflict if they are within $20 \mathrm{~m}$ distance. Unless specified separately, we assume two mobile groups of 10 nodes each and 20 channels; channels have uniformed bandwidth of 1 and each vehicle has backlogged traffic.

We focus on the scenarios with two mobile groups, initially well-separated. One mobile group will travel towards the other at a speed of $v \mathrm{~km} / \mathrm{h}$. When mobile groups move across each other, nodes from different groups will conflict with each other until they become wellseparated. We focus on investigating the performance when groups conflict. We divide time into small slots of $170 \mathrm{~ms}$, so that movements in each slot will not lead to significant changes in network topology. We examine the system performance at the slot level.
We compare the proposed approach to the conventional decentralized coordination approach proposed in [3]. We use the following performance metrics:

- System utility. We examine three utility functions: proportional fairness (Proportional), minimum node channel usage (MIN), and total channel usage (SUM). The proportional fair utility is calculated as $\sqrt[N]{\prod_{n=0}^{N-1} T P(n)}$ where $N$ is the number of nodes, and $\operatorname{TP}(n)$ represents the number of channels assigned to node $n$.

- Coordination overhead. We examine the total message exchange averaged over the time slots that groups conflict. Two devices coordinate by exchanging four messages. For the proposed approach, we also include the overhead of group-level spectrum usage broadcast.

It should be noted that our simulations do not implement the actual message exchange. Hence the system utilities do not include the impact of control overhead. This allows us to examine the system utility and overhead separately without delving into the detailed message exchange protocols and settings. We plan to implement these protocols and investigate their impact in a more detailed study.

\section{B. Experimental Results}

1) Comparison of different coordination approaches. We start by comparing the proposed hierarchical coordination system to the conventional local bargaining system. The convention system performs a network-wide coordination among all the affected users to approach a new assignment. Devices will interact with their peers (from the same and different groups) to adjust channel usage, and repeat the same process every time the system conflict condition changes.

Figure 4 plots the communication overhead for a sample deployment. At time slot 5, two groups are close to each other and some devices start to interfere. In each time slot, network-wide conflict condition changes and the conventional approach triggers negotiations. On the other hand, the proposed approach significantly reduces the overhead, to almost negligible. Only at time slot 5 and 58, we see a small amount of messages due to inter-group negotiations, and intra-group coordinations to refine individual assignments after mapping. However, the tradeoff to the decrease in overhead is the degraded system utility. Figure 5 plots the system proportional fair utility in each time slot, where the proposed approach leads to a noticeable degradation, roughly $30 \%$. We argue 


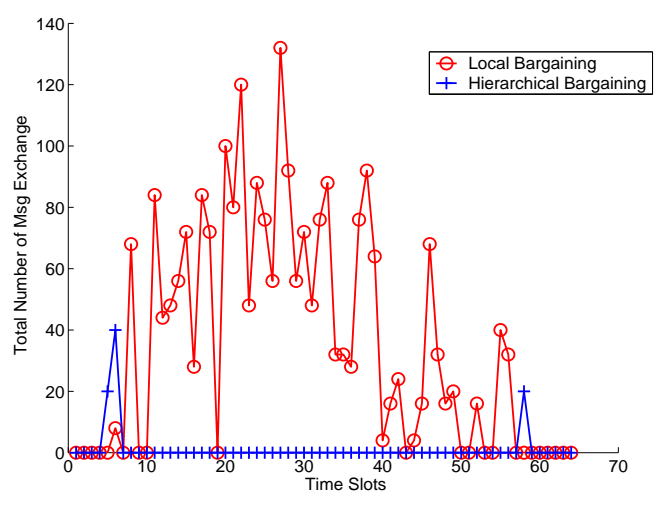

Fig. 4. Coordination overhead of the conventional coordination approach (marked as Local Bargaining) and the proposed hierarchical approach, assuming 2 mobile groups traveling at $30 \mathrm{~km} / \mathrm{h}$.

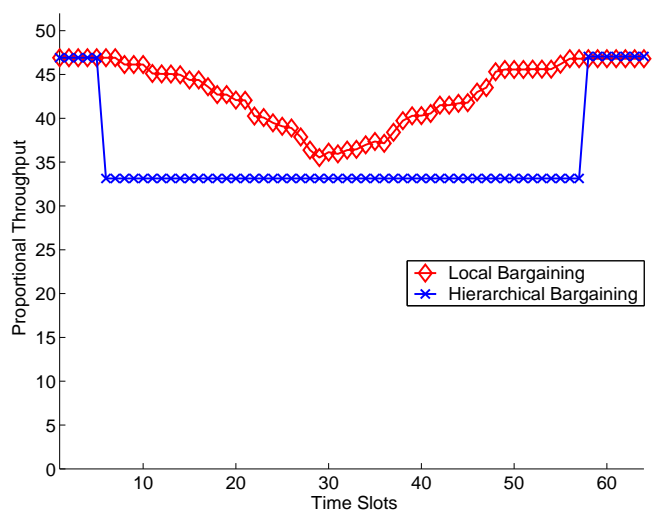

Fig. 5. System utility (proportional fair) of the conventional coordination approach (marked as Local Bargaining) and the proposed hierarchical approach, assuming 2 mobile groups traveling at $30 \mathrm{~km} / \mathrm{h}$.

that this tradeoff is reasonable because of the significant reduction in coordination overhead.

2) Impact of mobility speed. We evaluate the performance by varying mobile speed from $\{3 \mathrm{~km} / \mathrm{h}, 30 \mathrm{~km} / \mathrm{h}$, $60 \mathrm{~km} / \mathrm{h}$, to $120 \mathrm{~km} / \mathrm{h}\}$. We choose these values to examine the impact of topology variations in each time slot, and they should not be used to interpret real-life physical movements. Figure 6 and 7 compare the average communication overhead and system utilities of different approaches. For the conventional approach, we observe a linear increase of coordination overhead with the mobile speed, while the proposed system is insensitive.

3) Impact of network size. We examine the system performance at various network sizes while maintaining the same node density. Figure 8 and 9 plot the message overhead and system utilities, assuming a moving speed of $60 \mathrm{~km} / \mathrm{h}$. In the convention system, the coordination overhead scales linearly with the network size, while

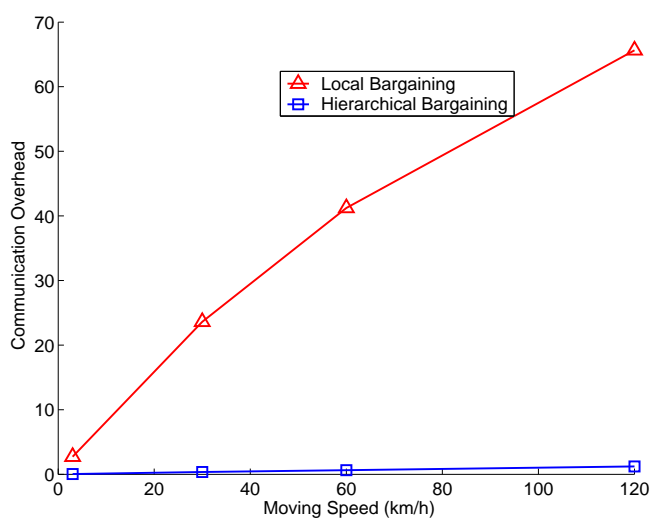

Fig. 6. Average coordination overhead of the conventional coordination approach (marked as Local Bargaining) and the proposed hierarchical approach, assuming 2 mobile groups traveling at various speeds.

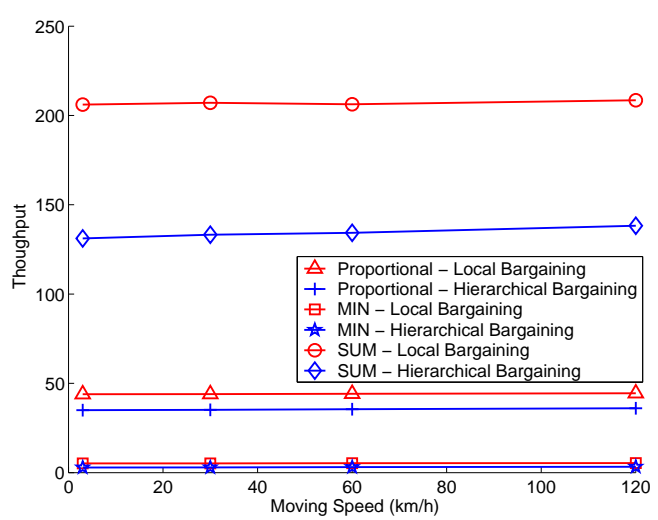

Fig. 7. Average system utilities of the conventional coordination approach (marked as Local Bargaining) and the proposed hierarchical approach, assuming 2 mobile groups traveling at various speeds.

the proposed approach maintains a very low overhead. Similar to earlier results, this reduction of complexity and overhead also results in 10-30\% utility degradation.

\section{CONClusion AND Future Work}

In this paper, we present a hierarchical coordination approach to manage spectrum usage for military networks with group mobility patterns. High mobility results fast and frequent changes in interference conditions, making the application of conventional coordination approaches challenging. Our approach makes use of group mobility patterns and focuses on reducing algorithm complexity and coordination overhead.

We propose a hierarchial spectrum allocation approach to distribute spectrum channels among mobile combat vehicles. When their movements lead to variations in conflict conditions, vehicles exercise a novel distributed approach based on inter-group and intra-group coordi- 


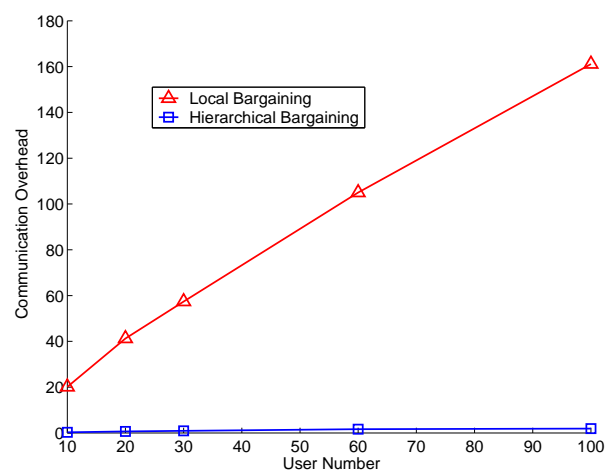

Fig. 8. Average coordination overhead of the conventional coordination approach (marked as Local Bargaining) and the proposed hierarchical approach, assuming 2 mobile groups of various sizes.

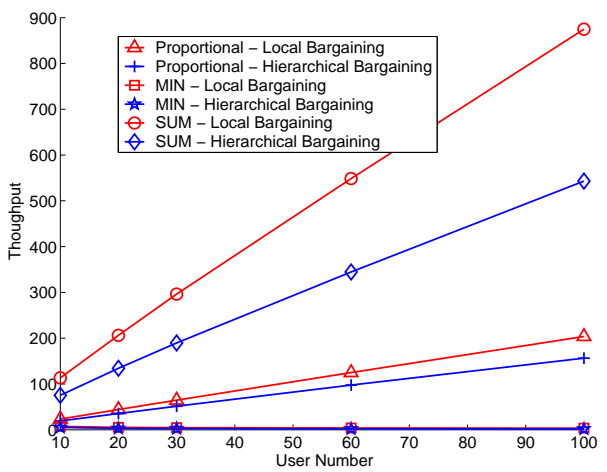

Fig. 9. Average system utilities of the conventional coordination approach (marked as Local Bargaining) and the proposed hierarchical approach, assuming 2 mobile groups of various sizes.

nations. Through this hierarchical design, we limit the majority of devices to only interact with their peers from the same group. Since each group has a relatively stable topology, our approach significantly reduces the coordination overhead. Experimental results show that the proposed approach can reduce the overhead by $10+$ folds while maintaining a slightly degraded system performance.

Our work provides an initial study of spectrum management in highly mobile networks with group mobility patterns. While our initial results on a simple network scenario are encouraging, there are many open problems to be addressed. First, the system utility depends heavily on the mechanisms to partition spectrum channels among conflicting groups. Our work uses a simple heuristics to equalize the average group poverty-line. While simple to implement, this mechanism leads to a $20-30 \%$ utility degradation. We are currently investigating new mechanisms to reduce the performance gap and support different types of applications.
Second, our work hasn't considered the impact of MAC and routing protocols as well as the radio propagation conditions. We are currently addressing this problem by building a system integrating various protocol components and examining the application level performance under realistic mobility patterns and channel models. This study will provide additional insights on the tradeoffs between coordination overhead and spectrum utilization.

\section{REFERENCES}

[1] Alicherry, M., Bhatia, R., AND Li, L. E. Joint channel assignment and routing for throughput optimization in multiradio wireless mesh networks. In Proc. of MobiCom (August 2005).

[2] Blakely, K., AND LoweKamp, B. A structured group mobility model for the simulation of mobile ad hoc networks. In Proc. of ACM MOBIWAC (2004).

[3] CAO, L., AND Zheng, H. Distributed spectrum allocation via local bargaining. In Proc. of SECON (Santa Clara, CA, September 2005).

[4] Hong, X., Gerla, M., Pei, G., and Chiang, C. A group mobility model for ad hoc wireless networks. In Proc. of ACM/IEEE MSWIM'99 (Seattle, WA, August 1999).

[5] Jain, K., Padhye, J., V.N.Padmanabha, And QIU, L. Impact of interference on multi-hop wireless network performance. In Proc. of MobiCom (San Diego, CA, September 2003).

[6] LiU, X., AND XiaO, H. Exploring opportunistic spectrum availability in wireless communication networks. In Proc. of VTC 2005.

[7] Nandagopal, T., Kim, T., Gao, X., and Bharghavan, V. Achieving MAC layer fairness in wireless packet networks. In Proc. of MobiCom (Boston, MA, August 2000).

[8] NiE, N., And Comaniciu, C. Adaptive channel allocation spectrum etiquette for cognitive radio networks. In Proc. of IEEE DYSPAN (November 2005).

[9] Peng, C., Zheng, H., And Zhao, B. Y. Utilization and fairness in spectrum assignemnt for opportunistic spectrum access. ACM Mobile Networks and Applications (MONET) (2006). to appear.

[10] Raychaudhuri, D., And Jing, X. A spectrum etiquette protocol for efficient coordination of radio devices in unlicensed bands. In Proc. of IEEE PIMRC (Sept. 2003).

[11] Wu, S. L., Lin, C. Y., Tseng, Y. C., And Sheu, J. P. A new multi-channel (MAC) protocol with on-demand channel assignment for multi-hop mobile ad hoc networks. In Proc. of I-SPAN (2000), pp. 232-237.

[12] Zheng, H., AND CAO, L. Device-centric spectrum management. In Proc. of IEEE DySPAN (Baltimore, MD, November 2005).

[13] Zheng, H., And Peng, C. Collaboration and fairness in opportunistic spectrum access. In Proc. of ICC (Seoul, Korea, June 2005).

[14] Zhou, B., Xu, K., And Gerla, M. Group and swarm mobility models for ad hoc network scenarios using virtual tracks. In Proc. of Milcom (2004). 